\title{
Discutindo a acessibilidade e usabilidade dos objetos de aprendizagem na plataforma OBAMA
}

\author{
Flávia Roldan Viana ${ }^{1}$, Layse Kelly Santos da Silva ${ }^{2}$, Ivanilka Lima de Azevedo ${ }^{3}$, \\ João Frederico Roldan Viana ${ }^{4}$ \\ ${ }^{1}$ Departamento de Práticas Educacionais e Currículo \\ Universidade Federal do Rio Grande do Norte - Natal, RN - Brazil. \\ ${ }^{2}$ Graduanda em Pedagogia \\ Universidade Federal do Rio Grande do Norte - Natal, RN - Brazil \\ ${ }^{3}$ Instituto Metrópole Digital \\ Universidade Federal do Rio Grande do Norte - Natal, RN - Brazil \\ ${ }^{4}$ Departamento de Computação \\ Faculdade Lourenço Filho - Fortaleza, CE - Brazil \\ flaviarviana.ufrn@gmail.com, laysekellyufrn@gmail.com, \\ ivanilkaazevedo@yahoo.com, jfredrv@gmail.com
}

\begin{abstract}
This paper investigates the accessibility and usability of learning objects that address the content of the Information Handling for the Initial Years and that are available in the OBAMA repository, from the criteria for evaluation of educational software of the Learning Object Review Instrument version 2.0 [NESBIT, BELFER, LEACOCK, 2009]. The results point to aspects to be observed by the teams of designers and programmers for the development of accessible $O A$.
\end{abstract}

Resumo. Este artigo investiga a acessibilidade e usabilidade dos Objetos de Aprendizagem que abordam o conteúdo do Tratamento da Informação para os Anos Iniciais e que estão disponíveis no repositório do OBAMA, a partir dos critérios para avaliação de softwares educacionais do Learning Object Review Instrument - versão 2.0 [NESBIT, BELFER, LEACOCK, 2009]. Os resultados apontam aspectos a serem observados pelas equipes de projetistas e programadores para o desenvolvimento de OA acessíveis.

\section{Introdução}

Vivemos o advento da globalização e a adoção em um novo estilo de vida que impõe o consumo das Tecnologias Digitais de Informação e Comunicação (TDIC), assim como nos demais campos da vida, tais tecnologias apresentam excelentes potencialidades ao processo de ensino e de aprendizagem, prenunciando novas propostas didáticometodológicas, que estimulem os estudantes a interagirem com a própria aprendizagem. E no contexto do ensino de matemática essas interações precisam ser enérgicas que nos levem a vivenciar, interpretar, abstrair, conhecimentos cada vez mais dinâmicos. 
VII Congresso Brasileiro de Informática na Educação (CBIE 2018)

Anais do XXIV Workshop de Informática na Escola (WIE 2018)

Diante dessas potencialidades Batista et al. (2017) enfatizam que os recursos digitais oferecem excelentes contribuições para um ensino de matemático em um contexto significativo, inovador em uma perspectiva de colaboração, protagonismo e implicação do aprendiz com sua trajetória formativa. Ainda no contexto das ferramentas digitais, estes pesquisadores relatam o desconhecimento de muitos professores dos objetos de aprendizagem (OA) e sua funcionalidade para introdução e sistematização de conceitos e conhecimentos matemáticos.

Considerando a necessidade da divulgação dos OA e visando ainda fomentar a reflexão sobre a prática docente na perspectiva de uma abordagem das TDIC, foi idealizado e implementado o repositório de Objetos de Aprendizagem para Matemática (OBAMA). A versão inicial do OBAMA foi concebida pelo Grupo de Pesquisa Matemática e Ensino - MAES da Universidade Estadual do Ceará - UECE, e atualmente é vinculado ao Grupo Interdisciplinar de Estudos e Pesquisas em Informática na Educação (GIIfE/UFRN). O objetivo maior do repositório, conforme Batista et al. (2017, p. 301) é

por meio de um trabalho interdisciplinar, procura combinar a visão de
profissionais da Educação com a de profissionais da área de Tecnologia da
Informação (TI) para o desenvolvimento de tecnologias educacionais. Assim,
o OBAMA contou com elementos de design e interação aliados a finalidade
pedagógica para a reconstrução do repositório OBAMA.

De modo prático, O OBAMA, se constituiu em uma plataforma que disponibiliza OA para matemática, categorizados por tema curricular, níveis de ensino da Educação Básica e descritores da prova Brasil, de modo a facilitar a busca e acesso por parte dos docentes que pretendam inserir tais recursos no desenvolvimento de suas aulas e situações de ensino. Primando pela interação e reflexão sobre a prática docente, o OBAMA oferece como diferencial a funcionalidade de oportunizar que seus usuários submetam seus planos de aulas enfocado a utilização dos OA enquanto metodologia de ensino e consolidação de conhecimentos matemáticos. Tais produções (planos de aulas, projetos de ensino, entre outros) passarão pela avaliação dos membros de uma curadoria, e se aprovados serão publicados no repositório, contando ainda com a possibilidade de receberem a contribuição em uma perspectiva colaborativa dos curadores do repositório, favorecendo assim, a reflexão sobre a prática e o crescimento profissional dos sujeitos implicados no fazer docente.

Como o fazer docente é dinâmico, a reflexão sobre a prática de ensino nos inquieta a aperfeiçoamentos constantes, buscando o alcance resultados mais efetivos, o repositório contará com a implementação de uma série de funcionalidades que objetivam subsidiar cada vez mais o trabalho dos docentes. Nesse sentido, percebemos a necessidade de investigar a acessibilidade e usabilidade dos OA, por pessoas com deficiência. Nesse artigo apresentamos os resultados iniciais desta investigação. Investigamos os OA para os Anos Iniciais do Ensino Fundamental que abordam o conteúdo do Tratamento da Informação, que engloba a leitura de gráficos e tabelas e que estão disponíveis no repositório do OBAMA, a partir dos critérios para avaliação de softwares educacionais do Learning Object Review Instrument - versão 2.0 [NESBIT, BELFER, LEACOCK, 2009], buscando apontar aspectos a serem observados para o desenvolvimento de OA acessíveis. A escolha pelo tema curricular Tratamento da 
VII Congresso Brasileiro de Informática na Educação (CBIE 2018)

Anais do XXIV Workshop de Informática na Escola (WIE 2018)

Informação advém do fato dos pesquisadores envolvidos estarem colaborando com pesquisas envolvendo tal tema.

\section{Método}

Configura-se como uma pesquisa investigativa de abordagem qualitativa e paradigma interpretativo. Foi realizado, inicialmente, uma revisão bibliográfica narrativa [COOK et al., 1997], de caráter exploratório, com o objetivo de familiarização com o objeto de estudo e aprimoramento de ideias, conforme orienta Gil [2007]. Foi realizada uma busca inicial no banco de dissertações e teses da Coordenação e Aperfeiçoamento de Pessoal de Nível Superior - CAPES com a utilização dos descritores: Objetos de Aprendizagem; Acessibilidade; Avaliação, foram encontrados 17 trabalhos que circunscrevem a temática do presente artigo e subsidiaram teoricamente a discussão dos resultados. De uma forma geral as pesquisas discutem os requisitos de acessibilidade de OA destinados a pessoas com deficiência, avaliam OA com base nos critérios para avaliação de softwares educacionais do LORI (Learning Object Review Instrument) - versão 2.0 e coadunam com a ideia de que os recursos pedagógicos digitais acessíveis podem vir a ampliar a construção do conhecimento por pessoas com deficiência.

O Segundo passo metodológico envolveu a escolha dos OA no repositório OBAMA. De acordo com Wiley [2002], OA são recursos digitais disponibilizados na internet, de fácil alcance por qualquer usuário e que possam ser usados como suporte para o processo de ensino e aprendizagem. Optou-se por definir critérios de escolha dos OA. Dessa forma, os OA deveriam atender aos seguintes critérios: Tema curricular Tratamento da Informação e nível de ensino, os anos iniciais do Ensino Fundamental. Como resultado desta etapa foram encontrados oito OA. Entretanto, para este artigo apresentamos a avaliação e discussão de apenas quatro, investigados até o momento de submissão deste artigo. Os OA avaliados foram:

- Qual o número de bolinhas? (OA1) O OA configura-se como um jogo interativo, no qual o usuário (o estudante) deverá contar quantas bolinhas tem no lado esquerdo da tela e selecionar a opção correta dentre as opções no lado direito (FIGURA 1).

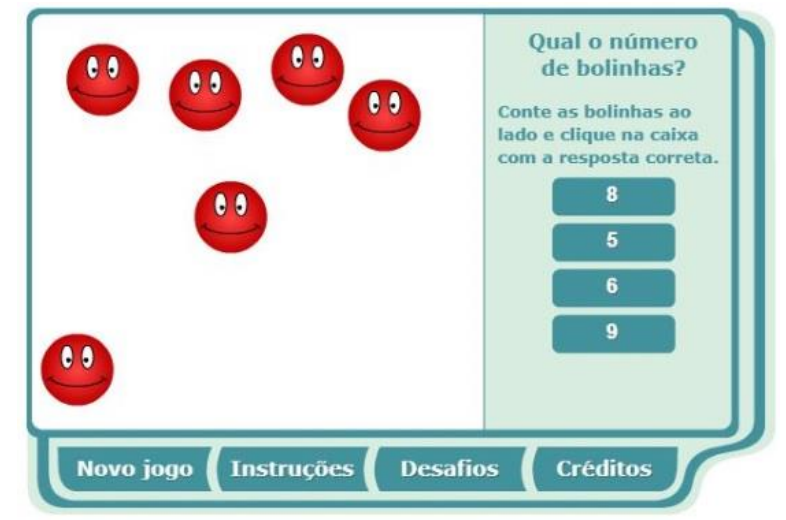

Figure 1. Captura da tela inicial do OA "Qual o número de bolinhas?" (http://mdmat.mat.ufrgs.br/anos_iniciais/objetos/bolinhas_contar.htm)

- Animais domésticos e silvestres (OA2). O OA é um jogo simples de classificação, no qual o estudante deverá classificar os animais em domésticos e silvestres para depois construir um gráfico (FIGURA 2). 
VII Congresso Brasileiro de Informática na Educação (CBIE 2018)

Anais do XXIV Workshop de Informática na Escola (WIE 2018)

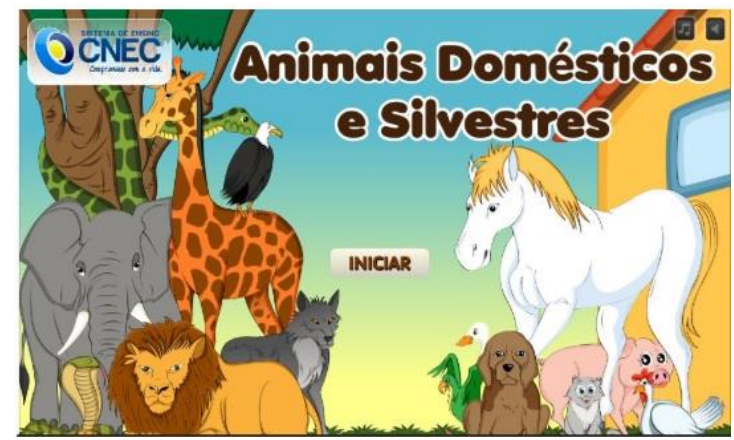

Figure 2. Captura da tela inicial do OA "Animais domésticos e silvestres" (http://www.noas.com.br/educacao-infantil/matematica/animais-domesticos-esilvestres/)

- Operando com números inteiros (AO3). Na atividade, os estudantes deverão preencher os campos de acordo com a representação do gráfico para realizer corretamente o cálculo (FIGURA 3).

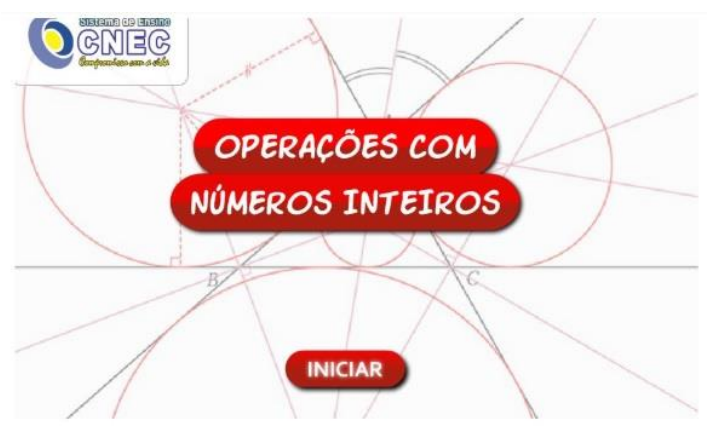

Figure 3. Captura da tela inicial do OA "Operações com números inteiros" (http://www.noas.com.br/ensino-fundamental-2/matematica/operacoes-comnumeros-inteiros/)

- Viajando com a matemática (OA4). O estudante nesta atividade poderá envolver-se com a aprendizagem de três temas: fuso horário, temperatura e saldo bancário (FIGURA $4)$.

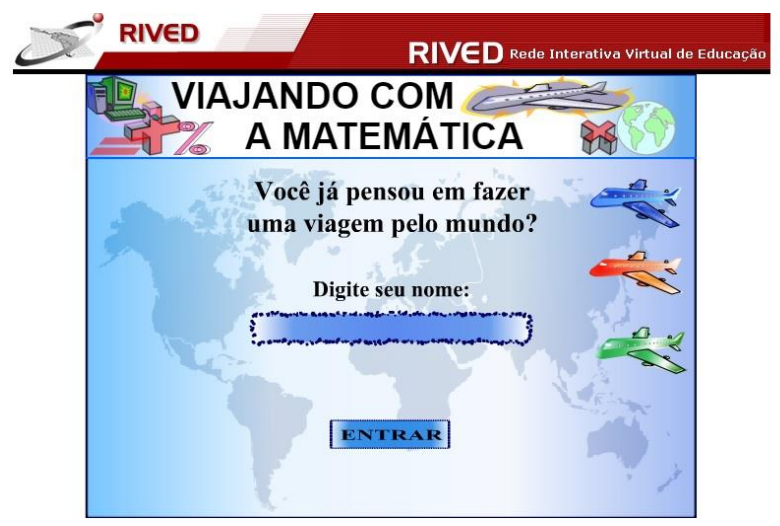

Figure 4. Captura da tela inicial do OA "Viajando com a matemática" (http://www.projetos.unijui.edu.br/matematica/fabrica_virtual/zeni_sidonia_fern ando/) 
VII Congresso Brasileiro de Informática na Educação (CBIE 2018)

Anais do XXIV Workshop de Informática na Escola (WIE 2018)

Após vivenciar na prática como os OA selecionados funcionam (vivência feita pelos próprios pesquisadores), foi selecionado o instrumento a ser utilizado para a avaliação dos mesmos. Dessa forma, optou-se analisar os OA com base nos critérios para avaliação de softwares educacionais do LORI (Learning Object Review Instrument) - versão 2.0 [NESBIT, BELFER, LEACOCK, 2009], por ser a metodologia de avaliação mais utilizada entre os estudos realizados na primeira etapa metodológica [MARZAL e PEDRAZZI, 2015; FERNANDEZ-PAPILLÓN, 2013; CESTEROS, 2014; GURER e YILDIRIM, 2014; e FENTON, 2014]. O LORI não foca especificamente na variável "acessibilidade", mas a inclui como uma das variáveis de avaliação.

De acordo com Nesbit, Belfer e Leacock (2009), o LORI (versão 2.0) é composto por nove variáveis de avaliação: qualidade de conteúdo, alinhamento das metas de aprendizagem, feedback e adaptação, motivação, design de apresentação, interação e usabilidade, acessibilidade, capacidade de reutilização e cumprimento das normas, que são avaliados por uma escala Likert de 1 (pior avaliação) à 5 pontos (melhor avaliação).

Na primeira variável, Qualidade do Conteúdo, a preocupação volta-se para uma apresentação equilibrada de ideias, com precisão e apropriado nível de detalhes. A segunda variável, alinhamento das metas de aprendizagem, refere-se a um alinhamento harmonioso entre atividades, avaliações e características do aluno. A terceira variável, analisa se o feedback e a adaptação foram e/ou estão sendo impulsionados pelo input ou modelação do aluno. A quarta variável, analisa a habilidade de motivar e interessar um grupo de alunos. A variável seguinte diz respeito ao design de apresentação, que traz uma análise da concepção de informações visuais e sonoras para uma aprendizagem reforçada e processamento mental eficaz. A sexta variável, interação e usabilidade, analisa se a navegação na interface do OA, a previsibilidade da interface do usuário e a qualidade das funções são fáceis de manusear. O sétimo critério de análise, avalia a acessibilidade do OA, checando a eficácia da usabilidade deste por usuários com algum tipo de deficiência, atentando ainda, se é possível o acesso por meio de diferentes tipos de dispositivos e em diferentes contextos. A capacidade de reutilização é a oitava variável e analisa o potencial do $\mathrm{OA}$ em ser utilizado em diferentes contextos. Por último o cumprimento das normas que se refere a avaliação dos campos de metadados associados ao OA, se estão completos e se seguem os padrões internacionais.

\section{Resultados e Discussão}

De acordo com as recomendações da $\mathrm{W} 3 \mathrm{C}$, para tornar acessível é necessário eliminar barreiras arquitetônicas, ofertar acesso físico e de comunicação, equipamentos e programas adequados, conteúdo e apresentação da informação em formatos alternativos. Todos os OA escolheram como ferramenta para o desenvolvimento o ambiente "Adobe

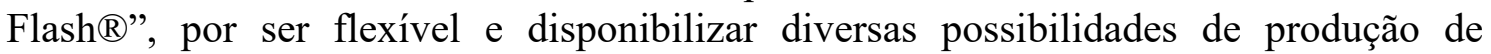
conteúdos interativos, que podem ser criadas ou importadas de outros aplicativos, tais como, vídeos, imagens, animações.

No OA1 (TABELA 1) foi observado que o mesmo está adequado quanto a sua classificação para o nível de ensino. Sua interface é limpa com comandos claros e objetivos. 
VII Congresso Brasileiro de Informática na Educação (CBIE 2018)

Anais do XXIV Workshop de Informática na Escola (WIE 2018)

Tabela 1. OA 1 - Qual o número de bolinhas?

\begin{tabular}{|c|c|c|c|c|c|c|}
\hline & \multicolumn{5}{|c|}{ escala Likert } & \\
\hline & 1 & 2 & 3 & 4 & 5 & NA \\
\hline 1. Qualidade do Conteúdo & & & & $\mathrm{x}$ & & \\
\hline 2. Alinhamento dos Objetivos de Aprendizagem & & & & & $\mathrm{x}$ & \\
\hline 3. Feedback e Adaptação & & & $\mathrm{x}$ & & & \\
\hline 4. Motivação & & & $\mathrm{x}$ & & & \\
\hline 5. Concepção (Design) da Apresentação & & $\mathrm{X}$ & & & & \\
\hline 6. Usabilidade Interativa & & & & & $\mathrm{x}$ & \\
\hline 7. Acessibilidade & $\mathrm{x}$ & & & & & \\
\hline 8. Reusabilidade & $\mathrm{x}$ & & & & & \\
\hline 9. Conformidade com os Padrões & & & $\mathrm{x}$ & & & \\
\hline
\end{tabular}

Entretanto, o jogo não apresenta desafios que provoquem no estudante a utilização de estratégias cognitivas que estimulem a criatividade e a conflitos de assimilação, acomodação e equilibração (conceitos piagetianos de esquemas ou estruturas cognitivas do pensamento). Ainda se observa que o jogo também não apresenta desafios crescente ou gradativo inerente ao conceito matemático apresentado: quantificação, pois a aba de desafios apesar de existir não funciona. Dessa forma, fica sempre no mesmo nível, variando apenas a quantidade de bolinhas. Há uma repetição de comando para início de uma nova etapa do jogo. Não apresenta informações sonoras, apenas visuais e simplificadas, sem detalhes ou instruções e não traz nenhum recurso de acessibilidade.

O OA2 (TABELA 2) é um jogo simples e possui um conteúdo claro, no qual cada nível está relacionado e depende do outro, não apresentando erros. Entretanto, nos aspectos visuais ele apresenta poucas informações sobre os animais e não tem comandos claros como, por exemplo, quando diz "pinte as molduras" não explica que, na verdade, o aluno tem que arrastar a cor do ícone que está na tela para o quadrado dos animais; as informações sonoras são escassas. Há pouca motivação do objeto de aprendizagem com o conteúdo, apenas no final do jogo faz relação com a tabela que é o objetivo de aprendizagem, mas não dá oportunidades para erros, o jogo é bem previsível, não deixando o erro acontece, ou seja, mesmo que o aluno "tente" selecionar a figura no lado errado do gráfico, ela não fixa e volta ao seu lugar inicial, indicado intrinsicamente que $o$ aluno errou e deve tentar novamente.

Tabela 2. OA 2 - Animais Domésticos e Silvestres

\begin{tabular}{|c|c|c|c|c|c|c|}
\hline & \multicolumn{5}{|c|}{ escala Likert } & \\
\hline & 1 & 2 & 3 & 4 & 5 & NA \\
\hline 1. Qualidade do Conteúdo & & & & $\mathrm{x}$ & & \\
\hline 2. Alinhamento dos Objetivos de Aprendizagem & & & & & $\mathrm{x}$ & \\
\hline 3. Feedback e Adaptação & & & & $\mathrm{x}$ & & \\
\hline 4. Motivação & & & $\mathrm{x}$ & & & \\
\hline 5. Concepção (Design) da Apresentação & & $\mathrm{X}$ & & & & \\
\hline 6. Usabilidade Interativa & & $\mathrm{X}$ & & & & \\
\hline 7. Acessibilidade & $\mathrm{x}$ & & & & & \\
\hline 8. Reusabilidade & $\mathrm{x}$ & & & & & \\
\hline 9. Conformidade com os Padrões & & & $\mathrm{x}$ & & & \\
\hline
\end{tabular}


VII Congresso Brasileiro de Informática na Educação (CBIE 2018)

Anais do XXIV Workshop de Informática na Escola (WIE 2018)

Outro ponto observado é que também não está acessível assim como o OA1. De acordo com Laplane e Batista (2008, p. 216),

o que determina o tipo de recurso a ser adotado é a necessidade, interesse,
disposição e objetivos do próprio sujeito, assim como as condições de
inserção sociocultural e familiar que tornarão um recurso mais adequado ou
viável que outro. Não há, portanto, uma conduta única que possa ser seguida
em todos os casos, mas sim, estratégias de caráter geral que podem facilitar o
trabalho escolar e derrubar barreiras de comunicação e acesso ao
conhecimento.

Dessa forma, para que um objeto de aprendizagem seja acessível, do ponto de vista inclusivo e educacional, é preciso disponibilizar aos usuários interfaces que respeitem suas especificidades de aprendizagem. Alguns documentos e estudos já orientam diretrizes de acessibilidade e usabilidade. A W3C organizou um guia internacional para a promoção de Acessibilidade da WCAG 2.0. A versão contempla 4 princípios: Percepção, Operação, Compreensão e Robustez.

O OA3 (TABELA 3) é um jogo com enunciados simples. Entretanto, algumas vezes pode aceitar ou gerar "erros", pois no mesmo campo pode aceitar outros números, o que pode confundir o aluno. Não há uma instrução de como realizar a soma ou subtração, o aluno precisaria de instruções externas, como a de um mediador, o que interfere diretamente em outros itens. Não possui nenhuma informação visual nem sonora. Não há gradação de níveis, apenas mudando as situações, variando soma de subtração. O feedback não estabelece relação com os objetivos do OA, que seria somar e subtrair utilizando números inteiros.

Tabela 3. OA 3 - Operando com números inteiros

\begin{tabular}{|c|c|c|c|c|c|c|}
\hline & \multicolumn{5}{|c|}{ escala Likert } & \\
\hline & 1 & 2 & 3 & 4 & 5 & NA \\
\hline 1. Qualidade do Conteúdo & & & $\mathrm{x}$ & & & \\
\hline 2. Alinhamento dos Objetivos de Aprendizagem & & & $\mathrm{x}$ & & & \\
\hline 3. Feedback e Adaptação & & & $\mathrm{x}$ & & & \\
\hline 4. Motivação & & & & $\mathrm{x}$ & & \\
\hline 5. Concepção (Design) da Apresentação & & $\mathrm{x}$ & & & & \\
\hline 6. Usabilidade Interativa & & & $\mathrm{x}$ & & & \\
\hline 7. Acessibilidade & $\mathrm{x}$ & & & & & \\
\hline 8. Reusabilidade & & $\mathrm{x}$ & & & & \\
\hline 9. Conformidade com os Padrões & & & $\mathrm{x}$ & & & \\
\hline
\end{tabular}

O OA3 também não apresenta acessibilidade para usuários com deficiência sensorial e/ou intelectual. De acordo com Torres e Mazzoni (2004, p. 152), "a nãoobservância dos princípios do design for all no espaço digital, pelos autores dos materiais disponibilizados nessa forma, pode ser considerada como uma discriminação feita a milhares de usuários".

Cada vez mais a acessibilidade e usabilidade são critérios essenciais na elaboração de OA, tendo em vista o aumento de usuários com deficiência na busca pelo uso desses recursos. Nielsen $(1993 ; 2005)$, em suas pesquisas, definiu 10 critérios a serem considerados para a concepção de interfaces: 1) Feedback; 2) Compatibilidade entre sistema e mundo real; 3) Controle pelo usuário; 4) Consistência; 5) Mensagens de erros adequadas; 6) Prevenção de erros; 7) Minimizar a sobrecarga de memória; 8) 
VII Congresso Brasileiro de Informática na Educação (CBIE 2018)

Anais do XXIV Workshop de Informática na Escola (WIE 2018)

Flexibilidade e eficiência; 9) Diálogos simples; e 10) Ajuda e documentação. Critérios esses não encontrados nos OA1, OA2, OA3 e OA4.

$\mathrm{Na}$ construção de objetos de aprendizagem acessíveis, deve-se ficar atento aos objetivos, que muitas vezes precisam ser redefinidos para adequar o conteúdo do OA à realidade.

No OA4 (TABELA 4), os objetivos do OA e de aprendizagem estão harmonizados aos conteúdos. O OA está sempre dando um feedback dos erros e acertos do usuário o que é positivo, pois assim ele sempre estará sabendo onde errou e tentando consertar, assim como os acertos. $\mathrm{O}$ aluno também pode obter bastante informações visuais, em todas as telas possui um botão de ajuda auxiliando na execução da atividade, além de fornecer outras informações necessárias para concluir as atividades, porém não possui informações sonoras.

O OA "Viajando com a matemática" (TABELA 4) não traz também nenhuma acessibilidade para o indivíduo com deficiência, seja ela sensorial e/ou intelectual.

Tabela 4. OA 4 - Viajando com a matemática

\begin{tabular}{|l|c|c|c|c|c|c|c|}
\hline & \multicolumn{5}{|c|}{ escala Likert } & \\
\hline & 1 & 2 & 3 & 4 & 5 & NA \\
\hline 1. Qualidade do Conteúdo & & & & & $\mathrm{x}$ & \\
\hline 2. Alinhamento dos Objetivos de Aprendizagem & & & & & $\mathrm{x}$ & \\
\hline 3. Feedback e Adaptação & & & & & $\mathrm{x}$ & \\
\hline 4. Motivação & & & & & $\mathrm{x}$ & \\
\hline 5. Concepção (Design) da Apresentação & & & & $\mathrm{x}$ & & \\
\hline 6. Usabilidade Interativa & & & & $\mathrm{x}$ & & \\
\hline 7. Acessibilidade & $\mathrm{x}$ & & & & & \\
\hline 8. Reusabilidade & & & $\mathrm{x}$ & & & \\
\hline 9. Conformidade com os Padrões & & & $\mathrm{x}$ & & & \\
\hline
\end{tabular}

\section{Considerações finais}

O foco da presente pesquisa foi investigar a acessibilidade e usabilidade dos OA, disponíveis no repositório OBAMA. Como descrito anteriormente, selecionou-se como objetos de pesquisa os OA que tratam do tema curricular - Tratamento da Informação para os anos iniciais do Ensino Fundamental. A relevância dessa pesquisa está em aprofundar estudos na área e aproximar o grupo de pesquisa, principalmente os projetistas de OA, dos princípios de acessibilidade e de usabilidade.

Os quatros objetos de aprendizagem avaliados estão escassos de acessibilidade, o que implica dizer que eles não podem ser usados por qualquer tipo de pessoa (com deficiência ou não), isso acaba restringindo o uso dos OA nos ambientes inclusivos e contextos de aprendizagem. Para que os OA avaliados tornem-se acessíveis faz-se necessário que todos os usuários tenham acesso às informações, logo, estas devem ser apresentadas de forma acessível. Vídeos, áudios ou informações sonoras, devem oferecer: audiodescrição para imagens, designer com cores contrastantes para habilitar o uso para pessoas com baixa visão, possuírem Legendagem para surdos e ensurdecidos (LSE) e/ou tradução/interpretação em Língua Brasileira de Sinais (Libras), e, de acordo com a proposta do Desenho Universal para o uso de suportes como tecnologias assistivas, os OA devem oferecer diversas opções de manipulação e execução, como, 
VII Congresso Brasileiro de Informática na Educação (CBIE 2018)

Anais do XXIV Workshop de Informática na Escola (WIE 2018)

por exemplo, o uso de apenas teclado virtual ou apenas o mouse, para pessoas com mobilidade reduzida ou deficiência física.

Outro aspecto observado é que as abordagens propostas, em sua maioria, não ofereceram níveis crescentes de dificuldades e desafios aos usuários, assim como a ausência de feedbacks e consequente suporte ao processo de construção da aprendizagem. Entendemos que a ausência de uma devolutiva satisfatória pode desencorajar o usuário, ao não os instigar a retornar para corrigir seus equívocos e consequente obter a melhoria sua performance.

Avaliamos que o designer utilizado na maioria dos OA não explora informações visuais e sonoras investindo no aspecto estético e interatividade da interface, aspectos estes que certamente despertaria o interesse do usuário favorecendo assim $o$ desenvolvimento de uma aprendizagem significativa disponível e acessível inclusive a pessoas com deficiência. Ao contrário disso, detectamos, no entanto, que alguns dos OA apresentaram uma interface que dificultavam a utilização de usuários experientes, o que inviabiliza a utilização dos tais em um contexto ensino inclusivo. Atentamos ainda, que dentre os OA analisados, a maioria não atende ao critério de utilização em contextos variados, incorrendo na característica de abordar os conteúdos de forma direta, pouco criativa e interativa.

Diante dos achados iniciais desta pesquisa, a ausência de OA acessíveis voltados para o ensino de matemática para os anos iniciais, ressaltamos a importância de aspectos que devem ser considerados a fim de atender a acessibilidade e usabilidade deste recurso de modo a favorecer a compreensão e aprendizagem, assim como possibilitar a inclusão de pessoas com deficiência em um ambiente interativo que favoreça uma aprendizagem significativa sob uma perspectiva lúdica.

Desse modo, considerando os Princípios de Design Universal", assim como as "Recomendações de Criação de Conteúdo Acessível para web" do World Wide Web Consortium (W3C) e as "Melhores Práticas para Produção de Aplicativos e de Conteúdo Acessível" apresentadas nas guias do Instructional Management Systems (IMS), os aspectos que favorecem a acessibilidade dos OA são: uso de imagens acompanhadas de textos alternativos, audiodescrição, emprego de cores com contrastes no design, de modo que tal funcionalidade possa ser habilitada para o uso por pessoas com deficiência visual (cegueira e baixa visão), não identificar palavras, por sua importância, controles independentes do dispositivo ou acessíveis via teclado utilizando cores, não conter muita informação, navegação fácil, entre outras.

Entretanto, é preciso considerar a acessibilidade desde o início da criação de um OA, para que não ocorram adaptações posteriores que não contemplem as especificidades de usabilidade por pessoas com deficiência.

$\mathrm{Na}$ continuidade deste trabalho, deverá ser aprofundado a análise dos OA focalizando os padrões e diretrizes de acessibilidade que os tornem acessíveis a usuários com deficiência, a elaboração de um instrumental de avaliação da acessibilidade e usabilidade e a organização de diretrizes para criação de conteúdo para OA acessíveis.

\section{Referências}

Batista, S. D., Carvalho, R. A. de., Oliveira, A. M. D. de., Silva, A. C. N., Oliveira, N. I. de and Maia, D. L. (2017) "OBAMA: um Repositório de Objetos de Aprendizagem para Matemática", In: VI Congresso Brasileiro de Informática na Educação (CBIE 
VII Congresso Brasileiro de Informática na Educação (CBIE 2018)

Anais do XXIV Workshop de Informática na Escola (WIE 2018)

2017), Anais dos Workshops do VI Congresso Brasileiro de Informática na Educação (WCBIE 2017), Recife: WCBIE 2017, p. 300-307.

Cesteros, A. M. (2014) Development of a Spanish Standard for Quality Assessment of Digital Educational Material. Tecnologias del Aprendizaje, IEEE Revista Iberoamericana, 9(4), 151-158.

Cook, D. J., Mulrow, C. D. and Haynes, R. B. (1997) Systematic reviews: synthesis of best evidence for clinical decisions. Annals of Internal Medicine, v.126, n.5, pp.376380 .

Fenton, G. (2014). Involving a young person in the development of a digital resource in nurse education. Nurse education in practice, 14(1), p. 49-54.

Fernández-Pampillón, A. M. (2013) A new AENOR project for measuring the quality of digital educational materials. Proceedings of the First International Conference on Technological Ecosystem for Enhancing Multiculturality (pp. 133-139). ACM.

Gil, A. C. (2007) “Didática do ensino superior”. São Paulo: Atlas, 2007

Gürer, M. D., and Yıldırım, Z. (2014) Effectiveness of Learning Objects in Primary School Social Studies Education: Achievement, Perceived Learning, Engagement and Usability. EGITIM VE BILIM-EDUCATION AND SCIENCE, 39(176), 131143.

Laplane, A. L. F and Batista, C. G. (2008) Ver, não ver e aprender: a participação de crianças com baixa visão e cegueira na escola. Caderno Cedes, Campinas, v. 28, n. 75 , p. 209-227.

Marzal, M. A., and Pedrazzi, S. (2015) Educational potential of topic maps and learning objects for m-learning in the knowledge society. Transinformação, 27(3), p. 229-244.

Nesbit, J. C., Belfer, K., and Leacock, T. (2009) "Learning object review instrument (LORI)". User Manual. E-Learning Research and Assessment Network (eLera) Portal for Online Objects in Learning (POOL), 2009. Disponível em: $<$ http://www.elera.net/eLera/Home/Articles/LORI\%201.5.pdf $>$. Acesso: 20 jun. 2018.

Nielsen, J. (1993) Usability Engineering. Boston, MA: Academic Press.

Nielsen, J. (2005) “Ten Usability Heuristics". Disponível em: $<$ http://www.useit.com/papers/heuristic/heuristic_list.html>. Acesso: 20 jun. 2018.

Torres, E. and Mazzoni, A. A. (2004) Conteúdos digitais multimídia: o foco na usabilidade e acessibilidade. Revista Ciência da Informação, v. 33, n. 2, p. 152 - 160.

W3C. Web Content Accessibility Guidelines 1.0. 1999. Dispível em: <http://www.w3.org/TR/WAI-WEBCONTENT/>. Acesso em: 28 agos. 2018.

Wiley, D. A. (2002) Connecting learning objects to instructional design theory: a defi nition, a metaphor, and a taxonomy. In: WILEY, David A. (Ed.). The instructional use of learning objects. Bloomington: AECT. Disponível em: http://reusability.org/read/. Acesso em: 02 jul. 2018. 